\title{
MATURITÉ SEXUELLE ET QUALITÉ DE LA COQUILLE DE L'CEUF
}

\author{
L. LACASSAGNE et I'. MONGIN \\ avec la collaboration technique de J. R. Acrite \\ Station de Recherches avicoles, \\ Centre national de Recherches zootechniques, Jouy-en-Josas (Seinte-et-Oise)
}

\section{SOMMAIRE}

Trois cents poulettes Leghorn blanche, nées le zo décembre $196_{3}$, furcut réparties, dès leur naissance, en 3 lots soumis à des régimes lumineux différents :

- . un lot témoin ' $\mathrm{C}$ élevé en lumière naturelle; 8 semaines ;

un lot A élevé en lumière naturelle avec un complément de lumière artiticielle jusqu à l'âge de

- un lot $\mathrm{B}$ élevé en lumière artificielle d’une durée quotidienne de 13 heures jusqu'à 8 semaines, puis de 7 heures jusqu'à la maturité sexuelle.

L'âge moyen à la ponte du premier ouf fut de I43,5 jours pour le lot témoin, I 5 I, I jours pour le lot $A$ et ${ }_{177}$, I jours pour le lot $B$ ce qui confirme les résultats déjà connus sur les variations de l'âge à la maturité sexuelle en fonction du régime d'éclairement au cours de la croissance.

Dans tous les lots la fréquence du nombre d'œufs à double jaune et d'œufs mous (sans membrane coquillière et à coquille peu calcifiée) diminue au cours des trois premiers mois de ponte.

La fréquence d'apparition de ces œufs anormaux est beaucoup plus grande dans le lot témoin à maturité sexuelle précoce que dans le lot $\mathrm{B}$ à maturité sexuelle retardée.

La qualité de la coquille des I 5 premiers œufs pondus par poule, qu'elle soit mesurée indirectement par le nombre d'œufs cassés ou fêlés, ou directement par l'évaluation de l'index de coquille, varie dans de grandes proportions en fonction de l'âge à la maturité sexuelle.

Ia solidité de la coquille est d'autant plus grande que la maturité sexuelle est plus tardive.

Au cours d'expériences antérieures nous avons comparé des lots de poulettes nées vers la mi-décembre puis élevées soit en lumière naturelle soit en lumière artificielle avec une durée d'éclairement de 6 heures par jour. Nous avons démontré que l'âge auquel la poulette entre en ponte agit directement sur la proportion d'œufs à double jaune ainsi que sur celle d'œufs sans membranes coquillières ou à coquilles incomplètes pondus durant les trois premiers mois de production. Le nombre de ces œufs anormaux est d'autant plus grand que la maturité sexuelle est précoce (I,AcAsSAGNE et JACQUET, Ig63-I965). 
Ces expériences ayant été effectuées sur des poules demi-lourdes Rhode-Island $\times$ Wyandotte, nous avons voulu vérifier si les mêmes phénomènes se retrouvaient chez la leghorn blanche et donner ainsi une portée plus générale aux résultats déjà acquis.

Nous avons en outre étendu nos contrôles à la qualité de la coquille de façon à vérifier si l'apparition d'œufs à coquilles incomplètes, signe le plus évident d'un dérèglement des derniers stades de l'ovogenèse, n'est pas le témoin de modifications plus générales des mécanismes présidant à la formation des coquilles.

\section{CONDITIONS EXPÉRIMENTALES}

Trois cents poulettes Leghorn blanches, nées le 20 décembre 1963 furent réparties au hasard, dès: leur naissance en 6 lots de $5^{\circ}$ animaux soumis deux à deux à des durées d'éclairement différentes.

\section{Du premier jour à la maturité sexuelle}

- Deux lots témoins $T_{1}$ et $T_{2}$ furent élevés en lumière naturelle.

- Deux lots traités $A_{1}$ et $A_{2}$ furent également élevés en lumière naturelle mais reçurent jusqu’à 8 semaines un complément de lumière artificielle pour leur fournir au total ${ }_{3} 3$ heures d'éclairement par jour.

- Deux lots traités $B_{1}$ et $B_{2}$ reçurent $\mathrm{I}_{3}$ heures de lumière artificielle jusqu'à l'âge de 8 semaines puis furent ramenés en une fois à une durée totale d'éclairement de 7 heures par jour (graph. I)

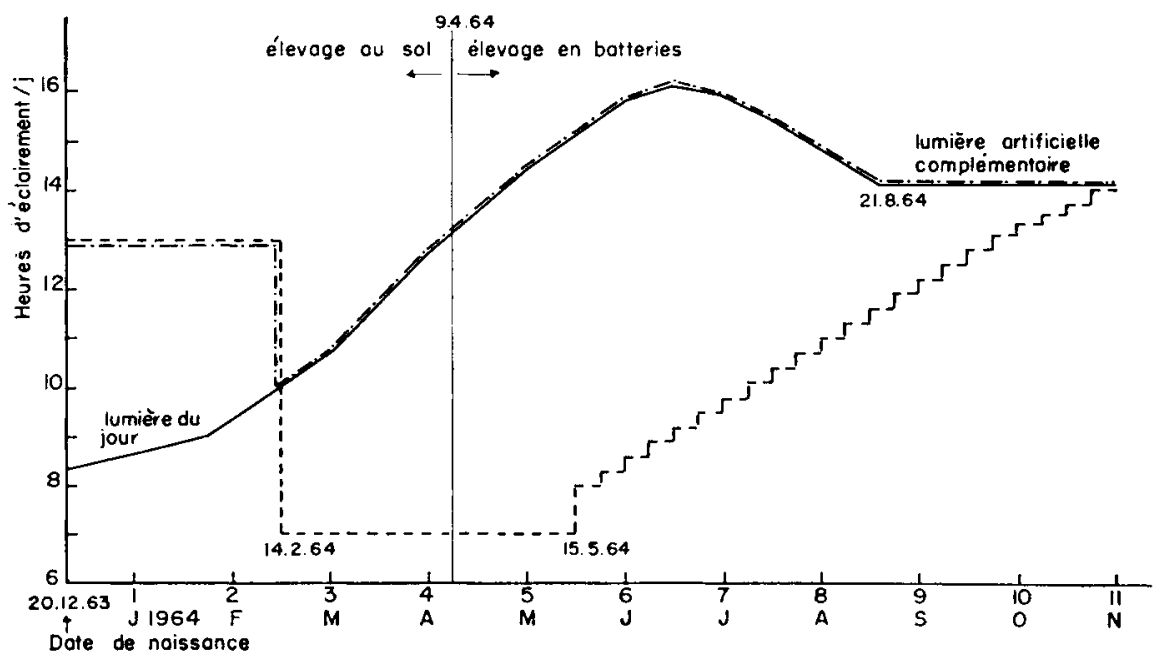

Légende : lot I

lot $\mathrm{A}$ - - - . -

lot $\mathrm{B}$

FIG. I. - Régimes lumineux expérimentés

Les lots $T_{1}, T_{2}, A_{1}, A_{2}$ furent élevés dans des poulaillers classiques, isolés et pourvus de fenêtres.

Les lots $B_{1}$ et $B_{2}$ furent élevés dans des bâtiments identiques, dépourvus de fenêtres et ventilés artificiellement.

A l'âge de 16 semaines, 40 poulettes furent prélevées au hasard dans chaque lot et transférées en batteries de ponte dans des bâtiments de même conception que ceux décrits plus haut.

A l'occasion de ce transfert chaque lot initial fut divisé en deux sous-groupes de manière à tenir compte, à l'analyse des résultats, d'éventuelles différences de milieu. Áinsi furent constitués I2 sous-groupes : $T_{1}$ donnant $T_{11}$ et $T_{12}, T_{2}$ donnant $T_{21}, T_{22} \ldots . B_{2}$ donnant $B_{21}, B_{22}$. 


\section{Après la maturité sexuelle}

- Les sous-groupes provenant des lots $T_{1}, T_{2}$ et $A_{1}, A_{2}$ reçurent un complément de lumière artificielle à partir du 2I août de manière à leur procurer au total It heures d'éclairement par jour.

- Les sous-groupes issus des lots $\mathrm{B}_{1}, \mathrm{~B}_{2}$ passèrent de 7 à 8 heures de lumière artificielle par jour le I 5 mai, à l'âge de 2 I semaines, puis furent soumis à une augmentation progressive de la durée d'éclairement à raison de $I_{5}$ minutes par semaine (graph. I).

Traitements expérimentaux

\begin{tabular}{|c|c|c|c|}
\hline $\begin{array}{c}\text { Lots de } 50 \text { poulettes } \\
\text { au sol. }\end{array}$ & $\mathrm{T}_{1}, \quad \mathrm{~T}_{2}$ & $A_{, 1} \quad A_{2}$ & $\mathrm{~B}_{1}, \quad \mathrm{~B}_{2}$ \\
\hline Bâtiments & Avec fenêtres & Avec fenêtres & Sans fenêtres \\
\hline $\begin{array}{l}\text { Jusqu'à l'âge de } \\
8 \text { semaines }\end{array}$ & Lumière du jour & $\begin{array}{c}13 \text { h de lumière au total : } \\
\text { lumière naturelle } \\
\text { plus lumière artificielle }\end{array}$ & $\begin{array}{l}13 \mathrm{~h} \text { de lumière } \\
\text { artificielle }\end{array}$ \\
\hline $\begin{array}{l}\text { De } 8 \text { semaines à } \\
16 \text { semaines }\end{array}$ & Lumière du jour & Lumière du jour & $\begin{array}{l}7 \mathrm{~h} \text { de lumière } \\
\text { artificielle }\end{array}$ \\
\hline à 16 semaines & & Passage en batteries & \\
\hline $\begin{array}{l}\text { Sous-groupes de } \\
20 \text { animaux }\end{array}$ & $\mathrm{T}_{11}, \mathrm{~T}_{12}-\mathrm{T}_{21}, \mathrm{~T}_{22}$ & $A_{11}, A_{12}-A_{21}, A_{22}$ & $\mathrm{~B}_{11}, \mathrm{~B}_{12}-\mathrm{B}_{21}, \mathrm{~B}_{22}$ \\
\hline $\begin{array}{c}\text { De } 16 \text { semaines à } \\
21 \text { semaines }\end{array}$ & Lumière du jour & Lumière du jour & $\begin{array}{l}7 \mathrm{~h} \text { de lumière } \\
\text { artificielle }\end{array}$ \\
\hline
\end{tabular}

Les poulettes furent nourries ad libitum durant toute la durée de l'expérience.

La qualité de la coquille des I 5 premiers œufs pondus par chaque poule fut évaluée à l'aide d'un index exprimant le poids de coquille pour une surface d'œuf de too centimètres carrés.

Chaque œuf fut cassé, les coquilles lavées et séchées à I 1 $0^{\circ} \mathrm{C}$ durant 24 heures puis pesées avec leurs membranes. La surface de l'cuuf fut déterminée par la formule de Muller et SCOTT (1940):

$$
\mathrm{S}\left(\mathrm{cm}^{2}\right)={ }_{4,67} \mathrm{P}^{2 / 3}(\mathrm{~g})
$$

et l'index exprimé par la formule suivante :

$$
\mathrm{I}=\frac{\text { Poids de coquille }+ \text { membranes }(\mathrm{g}) \times \text { roo }}{4,67 \mathrm{P}^{2 / 3}(\mathrm{~g})}
$$

Nous avons également enregistré pour chaque lot le nombre d'œufs mous, fêlés et cassés. Furent. considérés comme mous tous les oufs sans membranes coquillières ou munis de leurs membranes coquillières mais ne possédant que peu ou pas de dépôt calcique. Un œuf qui, après ramassage, paraissait trop abîmé pour être commercialisé était classé dans les "œuf cassés ". Si à l'exament une coquille présentait une ou plusieurs fêlures, l'œuf était classé comme "fêlé ".

Remarquons enfin que les batteries, d'un modèle courant, étant munies de grilles-planchers légèrement plus inclinées que la normale.

\section{RÉSUITATS}

\section{Maturité sexuelle}

I1 n'existe pas de différences significatives entre les sous-groupes des lots $\mathrm{A}$ $\left(A_{1}+A_{2}\right)$ et $B\left(B_{1}+B_{2}\right)$ mais le milieu a introduit une hétérogénéité dans les sousgroupes des lots $T\left(T_{1}+T_{2}\right)$. 
En comparant les moyennes générales des lots ' $\mathrm{C}$ et $\mathrm{A}$ (tabl. I) nous pouvons chiffrer à 8 jours le retard de maturité sexuelle apporté par une diminution de la durée quotidienne d'éclairement de $I_{3}$ heures à Io heures à l'âge de 8 semaines. De même il ressort une différence moyenne d'âge à maturité sexuelle de 34 jours entre les lots témoins T et les lots B élevés de 8 à $2 \mathrm{I}$ semaines avec une durée d'éclairement quotidienne de 7 heures.

Une analyse de variance sur les moyennes des sous-groupes nous montre que les différences observées sont significatives au seuil de $5 \mathrm{p}$. Ioo entre $\mathrm{T}$ et $\mathrm{A}$ et au seuil de 5 p. I ooo entre $T+A$ et $B$.

\section{Anomalies de l'ovogenèse: ceufs à double jaune, ceufs faiblement calcifiés, ceu/s sans membranes coquillières}

Dans tous les lots nous observons une évolution identique du nombre d'œufs mous et à double jaune durant les trois premiers mois de ponte (tabl. 2). La fréquence de ces anomalies est maximum durant le premier mois et diminue ensuite. Les niveaux sont cependant très différents d'un lot à l'autre. Sur les 3 mois nous observons en effet une fréquence d'apparition des œufs à double jaune et des oufs mous beaucoup plus faible dans les lots $B$ que dans les lots $T$. Ces résultats généralisent nos conclusions antérieures (animaux de souche différente) sur l'existence d'une liaison entre la maturité sexuelle et les anomalies de l'ovogenèse.

La similitude des résultats obtenus dans les lots ' $T$ ' et $A$, malgré une différence de maturité sexuelle de 8 jours constitue cependant une exception à laquelle nous ne pouvons pas pour l'instant fournir d'explication.

\section{Qualité de la coquille}

Io Qufs fêlés et cassés.

Le nombre d'œufs fêlés et cassés est considérablement plus élevé dans les lots T et $\mathrm{A}$ que dans les lots $\mathrm{B}$ puisque nous relevons respectivement les proportions de I6,0-I6,7 et 6,4 p. Ioo d'œufs cassés ou fêlés sur la production totale des trois premiers mois de ponte.

$2^{\circ}$ Index de coquille.

\section{A. Index moyen par poule.}

Les index moyens par sous-groupes sont réunis dans le tableau 3. Une analyse de variance ne fait pas apparaître de différence significative entre les différents sousgroupes à l'intérieur d'un même traitement ce qui prouve que le dispositif expérimental n'a pas introduit de distorsion dans la valeur des index. Nous obtenons ainsi un index moyen par poule de $6,83 \pm 0,05$ pour les lots $T$ et de $6,98 \pm 0,07$ et $7,66 \pm 0,06$ pour les lots $\mathrm{A}$ et $\mathrm{B}$ respectivement. Les différences entre les lots $\mathrm{T}$ et $\mathrm{B}$ d'une part et $A$ et $B$ d'autre part sont hautement significatives mais il n'y a pas de différence entre les lots $T$ et $\mathrm{A}$.

\section{B. Index moyen par ceuf.}

Les résultats obtenus pour chaque traitement sont reportés au graphique 2 . Nous observons une différence significative entre le lot $T$ (moyenne par œuf : $6,83 \pm$ 


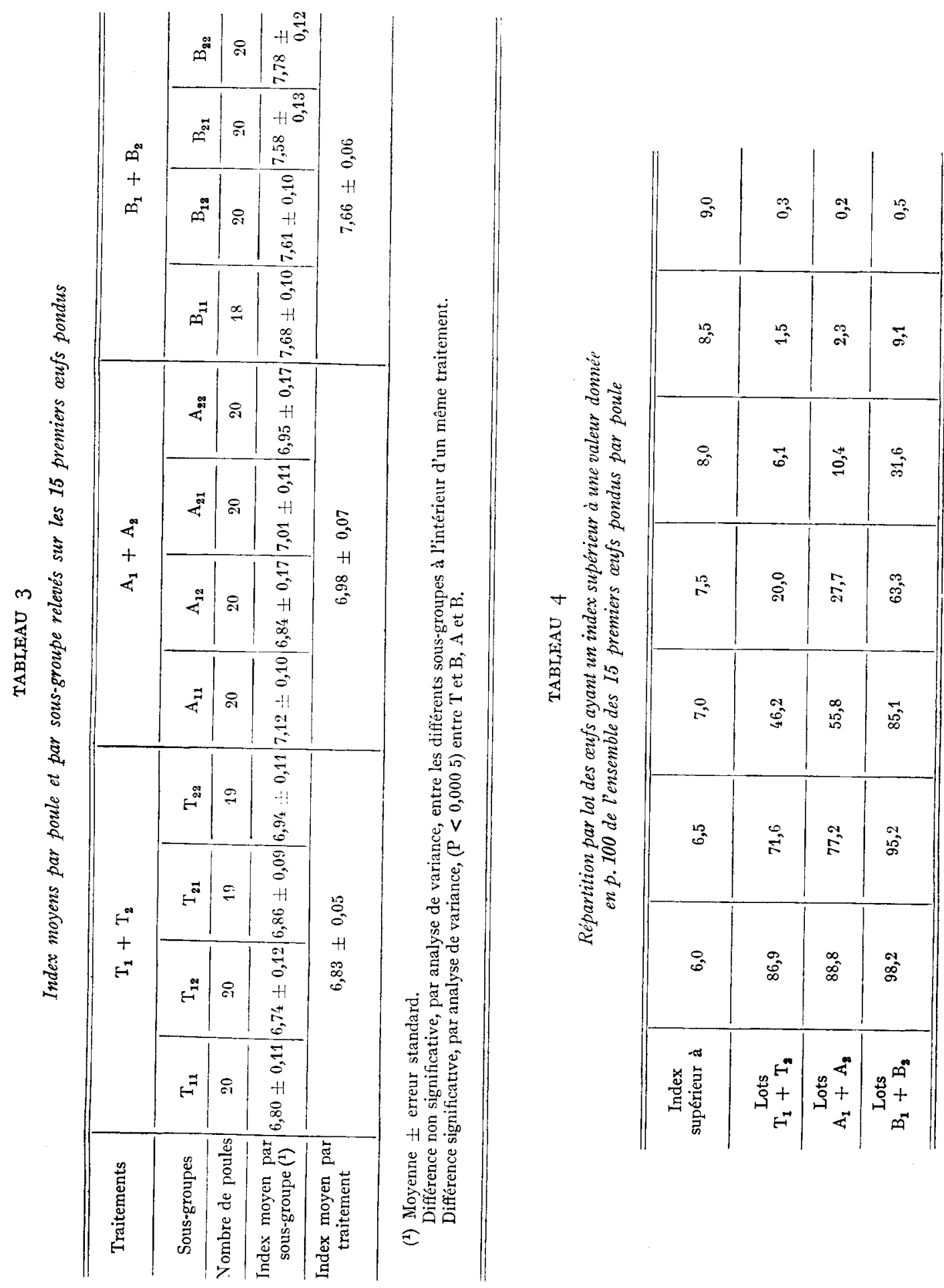


0,03 ) et le lot $\mathrm{A}$ (moyenne: $7,03 \pm 0,02$ ) et une différence hautement significative entre ces deux traitements et le lot $B$ (moyenne: 7,66 $\pm 0,02$ ). Les index de 6,837,03 et 7,66 correspondent respectivement à des épaisseurs de coquille de 297-305 et 333 millièmes de millimètre. Le regroupement des index par œuf fait donc apparaître une différence entre les lots $T$ et $A$ qui n'apparaissait pas dans l'analyse par poule.

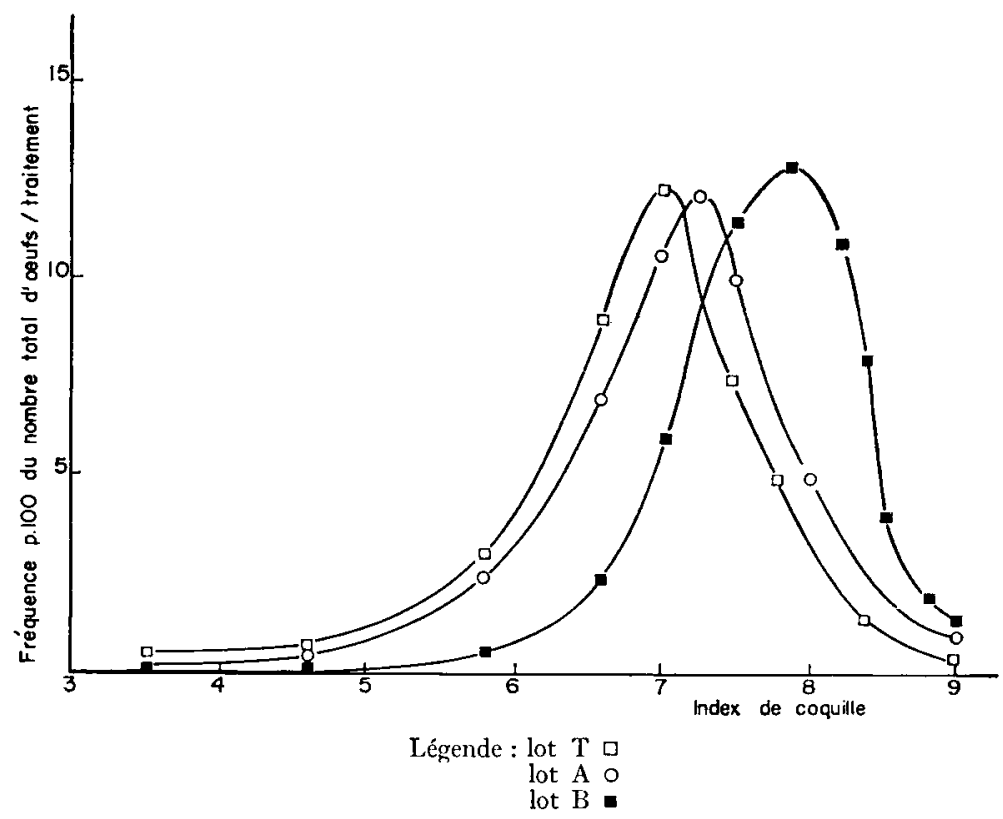

Fis. 2. - Fréquence, exprimée en p. 100, de lindex par nuts dans les lots $T$, $A$ et $B$

Les œufs pondus par les poulettes à maturité sexuelle retardée ont une qualité de coquille nettement supérieure à celle des autres lots. Une analyse détaillée des courbes de fréquence du graphique 2 nous révèle que $48 \mathrm{p}$. roo des œufs des lots $\mathrm{B}$ et Io $\mathrm{p}$. Ioo de ceux des lots $\mathrm{A}$ ont un index supérieur à celui des œufs des lots $\mathrm{T}$ élevés en lumière naturelle. Le regroupement des œufs en 7 classes (tabl. 4) donne une idée plus précise du phénomène.

L'ensemble de ces résultats recoupe sous un autre aspect les données du tableau 2 où figurent, par traitement, le nombre d'œufs cassés ou fêlés. A postériori on peut donc dire que plus le poids de coquille par unité de surface est élevé moins on observe de coquilles cassées ou fêlées.

\section{DISCUSSION}

On pourrait penser que les poulettes des lots B étant entrées en ponte un mois après les témoins ont eu la possibilité d'accumuler davantage de calcium dans leur squelette et bénéficier ainsi de réserves calciques beaucoup plus importantes en début de ponte; d'autant plus, qu'un aliment pondeuse a été distribué dès l'âge de $\mathrm{x} 6$ semaines dans tous les lots. 
En fait, il ne semble pas qu'il en soit ainsi. I'après Tay tor T. G. et Morris. 'T'. R. (I964) le poids du squelette à l'entrée en ponte de lots précoces ou tardifs est du même ordre de grandeur, mais les lots à maturité sexuelle précoce se trouvent dans l'incapacité de reconstituer leurs réserves calciques osseuses durant les premières semaines de production.

L'âge à la maturité sexuelle semble donc ètre le facteur essentiel dans l'apparition des différentes qualités de coquilles observées dans notre rapport. Les résultats obtenus dans cette expérience et nos résultats antérieurs (LACASSAGNE et JACQUET, I963, I965) qui mettent en évidence une relation entre l'âge à la maturité sexuelle et l'apparition d'anomalies de l'ovogenèse telle que cufs à double jaune ou sans coquilles, viennent à l'appui de cette hypothèse.

Rę̧ pour publication en septembre 1965.

\section{SUMMARY}

SEXUAL MATURI'T AND EGG SHLILL QUALI'Y

Three hundred White Leghorn pullets hatched on december 20 th $196_{3}$, were divided into three groups at hatching time and reared under various light treatments. 8 weeks.

- A group $A$ was reared under natural daylight with complementary artificial light up to

- A group 13 was reared up to 8 weeks under an artificial light treatment of ${ }_{3} 3$ hours per day, then of 7 hours up to sexual maturity.

2. The mean age of each group at first egg laying was 14.3.5 days, I5I.I days and I77.I days respectively for the control group, the $A$ group and the $I 3$ group, and thus asserts the results already known about the variations of age at sexual maturity proportionate to light treatment throughout growth.

3. In all groups, the incidence of double-yolked eggs, soft eggs (eggs without shell membranes or with an undercalcified shell) decreased throughout the first three months of lay.

The incidence of those abnormal eggs is much more frequent in the control group with early sexual maturity than in the group 13 with delayed sexual maturity.

4 The shell quality of the I 5 first eggs laid by hen, measured either indirectly according to the amount of broken or cracked eggs, or directly by the evaluation of the shell index, varies greatly with age at sexual maturity.

The more delayed sexual maturity, the greater is the shell strenght.

\section{RÉFÉRENCES BIBLIOGRAPHIQUES}

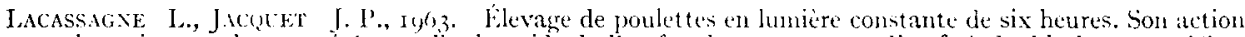
sur la croissance, la maturité sexuelle. le poids de l'ouf et le pourcentage d'oufs ì double jaune en début de ponte. Inn. Zootech., 12, $159^{-1} 72$.

Lacassagne L., Jacollet J. P’, ig65. Maturité sexuelle a apjarition d'aufs it double jaune cot sans coquille. Rôle du rẹgine lumineux et du niveau alimentaire. Inn. Zoolech., 14, 167-179

Mulber C. D., ScoTT II. M., I940. The porosity of egry shell on relation to hatchability. Poult. Sci., 19, I63-г66.

'TAYlor T.G., Morris T.R., igh. The eflect of earlyand late maturingron the squelet ton of pullets. World's Poult. Sci. J., 20, $294-20 \mathrm{j}$. 